\title{
artigo
}

Gerônimo, C.A.O.; Gomes, M.F.S.; Muniz, M.L.C.; Rocha, L.S.; Santos, M.S.T.G.; Galindo Neto, N.M.;

Especialização latu sensu de enfermagem em terapia intensiva no Brasil

\section{Especialização latu sensu de enfermagem em terapia intensiva no Brasil}

\author{
Latu sensu nursing specialization in intensive therapy in Brazil \\ Latu sensu enfermería especialización en terapia intensiva en Brasil
}

\begin{abstract}
RESUMO
Objetivo: caracterizar as especializações Latu Sensu de enfermagem em terapia intensiva no Brasil. Método: pesquisa documental, descritiva ocorrida com 501 matrizes curriculares de cursos de especialização de enfermagem em Terapia Intensiva. Resultados: observou-se predomínio de $494(98,60 \%)$ cursos ofertados por instituições privadas, $486(97,0 \%)$ na modalidade presencial, $271(54,20 \%)$ localizados nas capitais, $188(37,20 \%)$ no Sudeste e $137(27,34 \%)$ no Nordeste. Destacam-se os cursos com ênfase a área de Terapia Intensiva Geral/Adulto, representando 291 (58,08\%). Em 58 (11,58\%) as aulas ocorriam mensalmente e os temas relacionados ao Trabalho de Conclusão de Curso e à Gestão/gerenciamento foram os mais presentes, encontrados em $214(42,71 \%)$ e $203(40,52 \%)$, respectivamente. Conclusão: as especializações de enfermagem em terapia intensiva são ofertadas, em maioria, pela rede privada das regiões sudeste e nordeste, de forma presencial, com aulas mensais e os conteúdos acerca do trabalho de conclusão de curso e do gerenciamento são os mais encontrados.
\end{abstract}

DESCRITORES: Unidades de Terapia Intensiva; Bacharelado em Enfermagem; Educação de Pós-Graduação em Enfermagem; Enfermagem de Cuidados Críticos.

\section{ABSTRACT}

Objective: to characterize Latu Sensu nursing specializations in intensive care in Brazil. Method: documentary, descriptive research carried out with 501 curricular matrices of specialization courses in intensive care nursing. Results: there was a predominance of $494(98.60 \%)$ courses offered by private institutions, $486(97.0 \%)$ in person, $271(54.20 \%)$ located in the capitals, 188 $(37.20 \%)$ in Southeast and 137 (27.34\%) in the Northeast. The courses with emphasis on the General / Adult Intensive Care area stand out, representing $291(58.08 \%)$. In $58(11.58 \%)$ classes took place monthly and the topics related to Course Completion Work (TCC) and Management / management were the most present, found in $214(42.71 \%)$ and $203(40.52 \%)$, respectively. Conclusion: the nursing specializations in intensive care are offered, mostly, by the private network of the southeast and northeast regions, in person, with monthly classes and the contents about the course completion and management are the most found.

DESCRIPTORS: Intensive Care Units; Baccalaureate Nursing Education; Nursing Education Graduate; Critical Care Nursing.

\section{RESUMEN}

Objetivo: caracterizar las especializaciones de enfermería Latu Sensu en cuidados intensivos en Brasil. Método: investigación documental descriptiva realizada con 501 matrices curriculares de cursos de especialización en enfermería de cuidados intensivos. Resultados: hubo predominio de $494(98,60 \%)$ cursos impartidos por instituciones privadas, 486 (97,0\%) presenciales, $271(54,20 \%)$ ubicadas en las capitales, $188(37,20 \%)$ en Sureste y $137(27,34 \%)$ en el noreste. Destacan los cursos con énfasis en el área de Cuidados Intensivos Generales / Adultos, que representan 291 (58.08\%). En 58 (11,58\%) las clases se impartieron mensualmente y los temas relacionados con Trabajo de Finalización de Curso (CBT) y Gestión / gestión fueron los más presentes, encontrándose en $214(42,71 \%)$ y $203(40,52 \%)$, respectivamente. Conclusión: las especialidades de enfermería en cuidados intensivos son ofrecidas, en su mayoría, por la red privada de las regiones sureste y noreste, presencialmente, con clases mensuales y los contenidos sobre el trabajo de realización del curso y gestión son los más encontrados.

DESCRIPTORES: Unidades de Cuidados Intensivos; Bachillerato en Enfermería; Educación de Postgrado en Enfermería; Enfermería de Cuidados Críticos.

RECEBIDO EM: 15/03/2021 APROVADO EM: 31/03/2021 


\section{Caique Alberto de Oliveira Gerônimo}

Acadêmico do curso de Bacharelado em Enfermagem do Instituto Federal Educação, Ciência e Tecnologia de Pernambuco campus Pesqueira.

ORCID: 0000-0002-1933-1390

\section{Mariana Ferreira da Silva Gomes}

Acadêmica do curso de Bacharelado em Enfermagem do Instituto Federal Educação, Ciência e Tecnologia de Pernambuco campus Pesqueira.

ORCID: 0000-0003-4944-2339

\section{Marcela Lourene Correia Muniz}

Mestre em Educação para o Ensino da Saúde, Especialista em Nefrologia e em Gestão de Auditoria em Saúde. Professora do Instituto Federal Educação, Ciência e Tecnologia de Pernambuco - campus Abreu e Lima.

ORCID: 0000-0002-2279-533X

\section{Luanna dos Santos Rocha}

Mestre em Enfermagem, Especialista em Enfermagem Obstétrica. Professora do Instituto Federal Educação, Ciência e Tecnologia de Pernambuco - campus Pesqueira.

ORCID: 0000-0002-1013-5609

\section{Maria do Socorro Torres Galindo dos Santos}

Mestre em Ciências da Educação, especialista em Saúde Pública e em Docência do Ensino Superior, professora do curso de bacharelado do Instituto Federal Educação, Ciência e Tecnologia de Pernambuco - campus Pesqueira.

ORCID: 0000-0002-7893-0406

\section{Nelson Miguel Galindo Neto}

Doutor em Enfermagem, Mestre Enfermagem, Especialista em Didático-Pedagógica para o Ensino da Enfermagem, professor do curso de bacharelado do Instituto Federal Educação, Ciência e Tecnologia de Pernambuco - campus Pesqueira.

ORCID: 0000-0002-7003-165X

\section{INTRODUÇÃO}

A Unidade de Terapia Intensiva (UTI) é designada à prestação de assistência contínua aos pacientes em estado grave, com uso de tecnologias/ equipamentos necessários ao diagnóstico $\mathrm{e}$ tratamento. ${ }^{1} \mathrm{~A}$ alta complexidade dos cuidados nesse contexto, exige dos profissionais habilidades e competências específicas para uma atuação qualificada e resolutiva. ${ }^{2}$

Em conformidade com a Resolução nº7/2010 do Ministério da Saúde, o enfermeiro coordenador da equipe de enfermagem, pode assumir a responsabilidade técnica ou coordenação em, no máximo, duas UTI, deve ser intitulado, especialista em terapia intensiva ou outra especialidade destinada ao paciente crítico. Ademais, a equipe mínima da unidade deve conter pelo menos um enfermeiro assistencial para cada oito leitos ou fração, por turno. ${ }^{3-4}$

A complexidade da terapia intensiva exige dos profissionais assistência específica e qualificada, pois a condição crítica dos pacientes e a utilização de tecnologias demandam conhecimentos e habilidades para subsidiar a assistência prestada. ${ }^{2,5}$ Ao considerar que o profissional é influenciado pela sua formação, aponta-se a relevância de cursos de especialização que instrumentalizem a enfermagem para atuar nos cuidados intensivos.

Inseridos na modalidade de pós-graduação lato sensu, os cursos de especialização objetivam promover a qualificação profissional a partir do aprofundamento do conhecimento em uma área específica, ampliando por consequência as oportunidades no mercado de trabalho.6 Observa-se, pois, que a expansão da oferta de cursos de pós-graduação é uma realidade em todo país, existindo, entretanto, uma lacuna relacionada a publicações que tratem desse contexto. ${ }^{6-7}$

A caracterização das especializações de enfermagem em UTI possibilitará a identificação de lacunas, a compreensão de como encontra-se o cenário brasileiro e a distribuição de tais cursos nas variadas regióes do país. Essa carac- terização poderá contribuir com tomada de decisão acerca da estruturação dos cursos de pós-graduação de enfermagem e somar ao estado da arte acerca da temática.

Dessa forma, o estudo objetivou caracterizar as especializações Latu Sensu de enfermagem em terapia intensiva no Brasil.

\section{MÉTODO}

Esta pesquisa constitui-se de estudo descritivo, documental, quantitativo. A coleta ocorreu mediante acesso ao website do Ministério da Educação-MEC (emec.me.gov. br) para identificação das instituições de ensino que ofertavam as especializações. Posteriormente, o website de cada instituição foi acessado, para busca pelas informações acerca do curso de pós-graduação Latu Sensu de enfermagem em terapia Intensiva.

As matrizes curriculares dos cursos de especialização em UTI compuseram a população do estudo. Adotou-se como critério de inclusão o curso encontrar-se em 


\section{artigo}

Gerônimo, C.A.O.; Gomes, M.F.S.; Muniz, M.L.C.; Rocha, L.S.; Santos, M.S.T.G.; Galindo Neto, N.M.;

Especialização latu sensu de enfermagem em terapia intensiva no Brasil

status de funcionamento ativo perante o MEC e como critério de exclusão a indis-

ponibilidade de website das instituições para acesso as informações dos cursos.

Tabela 1 - Localização geográfica dos cursos de especialização em Enfermagem em Terapia Intensiva no Brasil, por região. Pesqueira, PE, Brasil, 2020

$\begin{array}{lccc}\text { REGIÃO } & \begin{array}{c}\text { LOCALIZADOS NO } \\ \text { INTERIOR N (\%) }\end{array} & \begin{array}{c}\text { LOCALIZADOS EM } \\ \text { CAPITAIS N (\%) }\end{array} & \text { TOTAL N (\%) } \\ \text { Sudeste } & 117(23,35 \%) & 71(14,17 \%) & 188(37,52 \%) \\ \text { Nordeste } & 55(10,98 \%) & 82(16,37 \%) & 137(27,34 \%) \\ \text { Sul } & 37(7,39 \%) & 35(6,99 \%) & 72(14,37 \%) \\ \text { Centro-Oeste } & 14(2,79 \%) & 42(8,38 \%) & 56(11,18 \%) \\ \text { Norte } & 6(1,2 \%) & 42(8,38 \%) & 48(9,58 \%) \\ \text { Nacional } & 229(45,71 \%) & 272(54,29 \%) & 501(100 \%) \\ \text { Fonte: }:-M E C, 2018 . & & & \end{array}$

Tabela 2 - Média de carga horária dos cursos de especialização em Enfermagem em Terapia Intensiva, no Brasil, conforme localização no interior ou capital, por região. Pesqueira, PE, Brasil, 2020

$\begin{array}{lcc}\text { REGIÃo } & \begin{array}{c}\text { CURSOS NO INTERIOR MÉDIA EM } \\ \text { HORAS }\end{array} & \begin{array}{c}\text { CURSOS NA CAPITAL MÉDIA EM } \\ \text { HORAS }\end{array} \\ \text { Sudeste } & 456,0 & 455,4 \\ \text { Nordeste } & 485,9 & 462,0 \\ \text { Sul } & 464,2 & 454,3 \\ \text { Centro-Oeste } & 503,5 & 476,5 \\ \text { Norte } & 510,0 & 436,6 \\ \text { Nacional } & 468,9 & 457,5 \\ \text { Fonte: e-MEC, 2018. } & & \end{array}$

Tabela 3 - Distribuição dos cursos de especialização em Enfermagem relacionadas a terapia intensiva no Brasil, de acordo com a área enfatizada. Pesqueira, PE, Brasil, 2020

ÁREA ENFATIZADA

$\mathrm{N}(\%)$

UTI geral/adulto $291(58,08 \%)$

UTI pediátrica e/ou neonatal

Urgência, emergência e UTI

$82(16,36 \%)$

$74(14,77 \%)$

UTI com ênfase em cardiologia

$16(3,19 \%)$

UTI adulto, pediátrico e neonatal

$16(3,19 \%)$

Obstetrícia e UTI neonatal

$10(1,99 \%)$

UTI e centro cirúrgico

$3(0,59 \%)$

Obstetrícia e UTI

$3(0,59 \%)$

UTI com ênfase em Gestão

$2(0,39 \%)$

UTI, urgência e emergência e centro cirúrgico

$2(0,39 \%)$

UTI com ênfase em neurologia

$1(0,19 \%)$

$1(0,19 \%)$
O instrumento de coleta utilizado foi construído especificamente para essa pesquisa e contemplou as variáveis disponíveis no site do MEC e nas matrizes curriculares dos cursos: nome e localização geográfica da instituição de ensino; categoria (privada ou pública); área enfatizada do curso; modalidade de oferta do curso (presencial, semipresencial ou à distância); periodicidade das aulas; carga horária total do curso e módulos/disciplinas ofertados.

Os dados foram coletados por três pesquisadores treinados para coleta e tabulação dos dados, no período de setembro e outubro de 2018 , sendo analisados posteriormente no Software $\mathrm{R}$, versão 3.5.1. mediante utilização de análise descritiva. Por esta pesquisa não envolver seres humanos e por utilizar dados de domínio público, não houve apreciação por Comitê de Ética em Pesquisa.

\section{RESULTADOS}

Foi identificado o quantitativo de 501 cursos de especialização em enfermagem relacionados a Unidade de Terapia Intensiva, cadastrados no e-MEC, e todos apresentaram websites disponíveis para levantamento dos dados (não havendo exclusão). Desses cursos, sete $(1,4 \%)$ eram oferecidos por instituições públicas e $494(98,6 \%)$ por instituições privadas.

No tocante à localização geográfica, observou-se discreta predominância de 272 (54,2\%) cursos ofertados nas capitais e que as regióes que mais possuíram tais cursos foram Sudeste e Nordeste, com 188 (37,52\%) e 137 (27,34\%), respectivamente. O detalhamento da localização geográfica dos cursos segue apresentado na tabela 1.

Em relação à modalidade em que os cursos eram ofertados, 486 (97,0\%) tratavam-se de cursos presenciais, 14 (2,8\%) de Educação à Distância (EAD) e um $(0,2 \%)$ semipresencial. Quanto à periodicidade das aulas, em $366(73,05 \%)$ cursos a informação não se encontrava disponível, em 58 (11,58\%) dos cursos as aulas ocorriam mensalmente, em 55 (10,98\%) quinzenalmente, em 15 (2,99\%) semanalmente, em sete $(1,40 \%)$ diariamente.

No tocante à carga horária, observou-se que os cursos tanto localizados no interior quanto nas capitais possuíram médias aproximadas, sendo a média geral nacional de 463,2 horas. Quando observadas as cargas 
horárias por região, a maior e menor média foi observada na região norte, de forma que os cursos localizados nos municípios de interior dessa região apresentaram média de 510 horas e os localizados nas capitais 436,6 horas, conforme detalhado na tabela 2 .

Dos 501 cursos analisados, 224 (44,71\%) disponibilizavam a matriz curricular online, nas quais observou-se a distribuição de conteúdos abordados a partir da leitura dos títulos das disciplinas/módulos. As disciplinas/conteúdos relacionados ao Trabalho de Conclusão de Curso (TCC) e à Gestão/gerenciamento foram as mais presentes, conforme detalhado na tabela 4 .

\section{DISCUSSÃO}

Observou-se que as regiões que possuíram mais cursos de especialização de enfermagem em Terapia Intensiva foram Sudeste e Nordeste. Tal fato pode ter relação com a distribuição desigual de leitos de UTI, por região do Brasil, uma vez que o Sudeste, com $51,9 \%$

Tabela 4 -Distribuição das disciplinas/conteúdos nos cursos de especialização em Enfermagem em Terapia Intensiva, no Brasil. Pesqueira, PE, Brasil, 2020

\section{DISCIPLINA/CONTEÚDO}

Trabalho de Conclusão de Curso

Gestão / Gerenciamento

Infectologia / Biossegurança

Sistematização da Assistência de Enfermagem

Ética e Bioética

Cardiologia

Farmacologia Intensiva

Assistência à vítima de agravo Clínico

Assistência à vítima de Trauma

Nefrologia

Ventilação Mecânica

Monitorização Cardíaca

Aspectos nutricionais em terapia intensiva

Exames Laboratoriais

Assistência Intensiva ao Queimado

Transplante / Captação de Órgãos

Pós-Operatório na terapia intensiva

Sepse

Oncologia

Fonte: websites das instituições de ensino, 2018. dos leitos, e o Nordeste com 20,7\%, consistem nas regiões que possuem mais leitos de terapia intensiva. ${ }^{8}$ Dessa forma, é possível que o quantitativo maior de leitos em tais regiões culmine na procura de enfermeiros dessas regiões por cursos de especialização, o que consiste em demanda que oportuniza a instalação desta especialização nestas regiões. Entretanto, diante da existência de UTI em todo território nacional, aponta-se que é relevante o maior investimento para que as demais regiões aumentem a oferta de especializações para enfermagem em terapia intensiva.

Ainda sobre a localização das instituições, observou-se um discreto predomínio dos cursos ofertados nas capitais. Esse achado pode ser justificado uma vez que os grandes centros e regióes metropolitanas concentram os estabelecimentos de saúde de alta complexidade e, logo, espera-se que possuam grande parte dos leitos de terapia intensiva. Dessa forma, compreende-se que a oferta das especializações em UTI ocorra em tais locais, diante da viabilidade para estágios/ práticas e visitas técnicas, bem como a oportunidade de empregos na área.

Houve destaque das instituições privadas na oferta das especializações em UTI voltadas para a enfermagem intensivista, o que converge com estudos acerca do perfil nacional da enfermagem, cujos resultados mostraram que a sua formação ocorre, predominantemente, em instituições particulares. ${ }^{9-10}$ Diante desse contexto, aponta-se relevância de as pesquisas acerca da formação em enfermagem não se limitem às instituições públicas, mas incluam a rede privada nos seus cenário de estudo, dada a proporção de enfermeiros que são graduados e pós-graduados por tais instituições.

No tocante à modalidade dos cursos, destacaram-se os presenciais. Tal fato é relevante e pertinente, diante do posicionamento político e pedagógico prevalente no cenário nacional de enfermagem que não coaduna com a formação EAD. A formação em enfermagem deve ser presencial para versar sobre a qualidade mediante possibilidade de inserção em cenários práticos que não são substituíveis por recursos tecnológicos virtuais. ${ }^{11}$

Em relação à periodicidade das aulas, a maioria das especializações em UTI apresentaram aulas ministradas mensalmente. Esse fato pode ocorrer uma vez que a elevada carga horária trabalhada e as escalas de plantão predispõem os profissionais a possuírem baixa disponibilidade para cursar a especialização, de forma que as instituições de ensino organizam os cronogramas com encontros mensais, para elevar a chance de adesão ao curso.

No que diz respeito as áreas enfatizadas, destaca-se predominância das especializações em terapia intensiva voltadas ao público adulto geral, fato coerente com a distribuição dos leitos de UTI no país, que aponta que $66,4 \%$ dos leitos do território nacional são destinados a população adulta. ${ }^{12}$ Justifica-se, pois, a maior demanda por profissionais capacitados na oferta de cuidados intensivos para a população adulta, quando comparada a outros ciclos de vida.

Dentre as disciplinas e conteúdos que integraram a matriz curricular das especializações em UTI (avaliadas a partir do seu título), destacou-se os referentes ao Trabalho de Conclusão de Curso. Tal fato é relevante uma vez que a inserção de conteúdos referentes à pesquisa 


\section{artigo}

Gerônimo, C.A.O.; Gomes, M.F.S.; Muniz, M.L.C.; Rocha, L.S.; Santos, M.S.T.G.; Galindo Neto, N.M.;

Especialização latu sensu de enfermagem em terapia intensiva no Brasil

nos cursos de pós-graduação corrobora para a adoção da Prática Baseada em Evidência (PBE) na atuação profissional da enfermagem. ${ }^{13}$ Cabe destacar que, como não foi realizada a leitura das ementas das disciplinas dos cursos, alguns conteúdos podem ter frequências maiores do que as referenciadas neste estudo. Isto pode ocorrer por tais conteúdos serem abordados de forma integrada a outros, bem como por se desenvolverem transversalmente entre disciplinas.

A limitação do estudo consistiu na análise ter ocorrido acerca dos cursos brasileiros, de forma que a especialização de enfermagem em terapia intensiva pode ter perfil distinto em outros países. Outro fator limitante diz respeito à pesquisa ter contemplado as matrizes curriculares dispo- níveis para acesso virtual, assim, as matrizes das especializações que não se encontravam disponíveis podem divergir dos resultados apresentados.

\section{CONCLUSÕES}

O estudo apontou que a maioria dos cursos de pós-graduação brasileiros são ofertados pela rede privada, distribuídos de forma equilíbrio de ofertas entre capitais e cidades do interior, como resultado do processo de expansibilidade e interiorização do ensino, assim como, da crescente demanda por especialização e qualificação profissional. Os cursos em sua maioria são na modalidade presencial, acompanhando o contexto político pedagógico nacional. Des- taca-se a predominância da oferta dos cursos na região Nordeste e Sudeste, onde surge uma lacuna relacionada ao percentual reduzido de oferta dos cursos na região Sul, Norte e Centro-Oeste. No que diz respeito aos conteúdos, aponta-se a necessidade de se investigar de que forma temáticas como ventilação mecânica, monitorização cardíaca e sepse são abordados nos cursos, visto que estes não foram explicitamente recorrentes nas grades curriculares analisadas, mesmo compreendendo-se que tais assumem grande importância no cotidiano do processo do cuidar em cenários de terapia intensiva, sendo sua não apropriação causa prejuízos na qualidade da assistência prestada pelos profissionais formados.

\section{REFERÊNCIAS}

1. Reisdorfer N, Hildebrandt LM, Leite MT. Enfermagem em unidade de terapia intensiva: atenção ao paciente com sintomatologia psiquiátrica. Rev Enferm UFSM [serial on Internet]. 2018 [cited 2021 mar 10]; 8(3):530-543. Available from: https://periodicos.ufsm.br/reufsm/article/view/28915

2. Oliveira NC de, Peçanha TBA, Fonseca C dos SG da. Desafios enfrentados pelos enfermeiros para garantir sua autonomia no cenário de alta complexidade. SaudColetiv (Barueri) [Serial on Internet]. 2020 [cited 2021 mar 29];9(50):1842-1850. Available from: https:/revistas. mpmcomunicacao.com.br/index.php/saudecoletiva/article/view/167

3. Ministério da Saúde (BR). Resolução n 7, de 24 de fevereiro de 2010. Dispõe sobre os requisitos mínimos para funcionamento de Unidades de Terapia Intensiva e dá outras providências. [serial on Internet]. 2010 [cited 2021 mar 10]. Available from: https:/bvsms.saude.gov.br/bvs/ saudelegis/anvisa/2010/res0007_24_02_2010.html

4. Santos MAB, Sá GGM, Caetano JÁ, Costa AB, Muniz MLC, Galindo Neto NM. Dissertações e teses da enfermagem brasileira acerca da unidade de terapia intensiva. Rev Rene [serial on Internet]. 2017 [cited 2021 mar 10]; 18(4):521-7. Available from: http://www.periodicos.ufc. br/rene/article/view/20256

5. Massaroli R, Martini KG, Massaroti A, Lazzari DD, Oliveira SN, Canever BP. Trabalho de enfermagem em unidade de terapia intensiva e sua interface com a sistematização da assistência. Esc Anna Nery [serial on Internet]. 2015 [cited 2021 mar 10];19(2):252-258. Available from: http:/ www.scielo.br/pdf/ean/v19n2/1414-8145-ean-19-02-0252.pdf

6. Fernandes JD, Silva RMO, LSR da Mota etal. Mapeamento dos cursos de especialização em enfermagem em sua totalidade e contradições. Rev enferm UFPE on line.[serial on Internet]. 2017 [cited 2021 mar 29];11(6):2459-65, Available from: https:/periodicos.ufpe.br/revistas/ revistaenfermagem/article/view/23410/19080

7. Fernandes JD, Silva RMO, Silva ACP, Mota LSR, Cordeiro ALOA, Souza RSA. Perfil dos cursos de especialização em enfermagem no município de Salvador, Bahia, Brasil. Rev. baiana enferm [serial on Internet]. 2017 [cited 2021 mar 29]; 31(2):e16660. Available from: https:// cienciasmedicasbiologicas.ufba.br/index.php/enfermagem/article/ view/16660/14624

8. Cotrim Junior DF, Cabral LMS. Crescimento dos leitos de UTI no país durante a pandemia de Covid-19: desigualdades entre o público x privado e iniquidades regionais. Physis [serial on Internet]. 2020 [cited 2021 mar 10]; 30(3): e300317. Available from: http://www.scielo.br/scielo. php?script=sci_arttext\&pid=S0103-73312020000300316\&tlng=pt

9. Machado MH, Wermelinger M, Vieira M, Oliveira E, Lemos W, Aguiar Filho WA, et al. Aspectos gerais da formação da enfermagem: o perfil da formação dos enfermeiros, técnicos e auxiliares. Enferm. Foco [serial on Internet]. 2016 [cited 2021 mar 10]; 6(2/4):15-34. Available from: http://revista.cofen.gov.br/index.php/enfermagem/article/viewFile/687/297

10. Frota MA, Wermelinger MCMW, Vieira LJES, Ximenes Neto FRG, Queiroz RSM, Amorim RF. Mapeando a formação do enfermeiro no Brasil: desafios para atuação em cenários complexos e globalizados. Ciênc. saúde coletiva [Serial on Internt]. 2020 [cited 2021 mar 29]; 25(1): 25-35. Available from: http:/www.scielo.br/scielo.php?script=sci_arttext\&pid=S1413-81232020000100025\&lng=en

11. Lira ALBC, Adamy EK, Teixeira E, Silva FV.Educação em enfermagem: desafios e perspectivas em tempos de pandemis COVID-19. Rev Bras Enferm [serial on Internet]. 2020 [cited 2021 mar 10]; 73(Suppl 2):e20200683. Available from: https:/www.scielo.br/pdf/reben/ v73s2/pt_0034-7167-reben-73-s2-e20200683.pdf

12. Associação de Medicina Intensiva Brasileira (AMIB). AMIB divulga primeira parte do censo 2016 com mapeamento das UTIs brasileiras. [serial on Internet]. 2017 [cited 2021 mar 12]. Available from: https:// www.amib.org.br/noticia/nid/amib-divulga-primeira-parte-do-censo-2016-com-mapeamento-das-utis-brasileiras/

13. Silva JOM, Santos LCO, Menezes NA, Lopes Neto A, Melo LS, Silva FJCP. Utilização da prática baseada em evidências por enfermeiros no serviço hospitalar. Cogitare Enferm [serial on Internet]. 2021 [cited 2021 mar 10]; 26:e67898. Available from: https://revistas.ufpr.br/cogitare/article/view/67898/pd 